\title{
Foetal heart rate variability frequency characteristics with respect to uterine contractions
}

\author{
Mario Cesarelli*, Maria Romano, Mariano Ruffo, Paolo Bifulco, Giulio Pasquariello
}

DIBET - University "Federico II”, Naples, Italy;

*Corresponding author.

Email: cesarell@unina.it; mariarom@unina.it; mariano.ruffo@libero.it; pabifulc@unina.it; giulio.pasquariello@unina.it

Received 6 August 2010; revised 9 September 2010; accepted 13 September 2010.

\begin{abstract}
Monitoring foetal health is important to appropriately plan pregnancy management and delivery. Cardiotocography (CTG) is one of the most employed diagnostic techniques. Because CTG interpretation still lacks of complete reliability, new methods of interpretation and parameters are necessary to further support physicians' decisions. To this aim, indexes related to variability of foetal heart rate (FHRV) are particularly studied. Frequency components of FHRV and their modifications can be analysed by applying a time-frequency approach, which allows for a distinct understanding of the spectral components related to foetal reactions to internal and external stimuli and their change over time. Being uterine contractions (UC) strong stimuli for the foetus and his autonomic nervous system (ANS), it is worth exploring the FHRV response to UC. This study analysed modifications of FHRV frequency characteristics with respect to $108 \mathrm{UC}$ (relative to 35 healthy foetuses). Results showed a statistically significant ( $t$-test, $p<$ 0.01 ) power increase of the FHRV in both $L F$ and HF bands in correspondence of the contractions. Moreover, we observed a shift to higher values of the maximum frequency contained in the signal corresponding to the power increase. Such modifications of the FHRV power spectrum can be a sign of ANS reaction and therefore represent additional, objective information about foetal reactivity and health during labour.
\end{abstract}

Keywords: Foetal Heart Rate, Uterine Contractions, Foetal Monitoring

\section{INTRODUCTION}

Cardiotocography (CTG) is one of the most diffused, non-invasive pre-natal diagnostic techniques, in clinical practice, to monitor foetal health, both in ante partum (third trimester of pregnancy) and intra partum period. It can be used from the $24^{\text {th }}$ week of gestation to delivery. However, in some countries, in clinical routine, it is generally used from the $35^{\text {th }}$ week and it is a medical report with legal value [1].

In CTG monitoring, foetal heart rate (FHR) and uterine contractions (UC) are simultaneously recorded by means of two probes placed on the maternal abdomen (a US Doppler probe for FHR signal and a pressure transducer for UC signal) [2].

Cardiotocographic data provide physicians information about healthy foetal development. To assess foetal health and reactivity, gynaecologists and obstetrics evaluate specific clinical signs (average value of FHR, number and kind of accelerations and decelerations in FHR signal, intensity, though as relative and not absolute values, and number of UC and their correlation with FHR modifications, etc). Important physiological mechanisms, like thermoregulatory oscillations, maturational changes with advancing gestational age, foetal behavioural states and maternal drugs can influence FHR patterns. In addition, clinicians generally make their evaluation on the basis of an eye inspection of cardiotocographic traces. The validity of the diagnostic procedure is hence still limited by the lack of complete objectivity and reproducibility. Moreover, even if CTG monitoring has been proved to be useful in early detection of foetal distress and, in intra partum period, electronic foetal monitoring led to a considerable reduction of mortality $[1,3,4]$, it is not been found a significant decrease of postnatal injuries, such as cerebral palsy [5]. Besides, some authors state that prenatal stress can provoke changes in foetal endocrine and metabolic processes that can impact the later health of children and adults [6] and that from oxygen deprivation during delivery, a rare but devastating event, lifelong disability can result [7].

Unfortunately, non-invasive methods to measure di- 
rectly the foetal acid-base status and cerebral oxygenation do not exist and clinicians have to rely upon indirect measures. Therefore, more detailed information about the foetal status is necessary and can be particularly useful during the last period of gestation and labour.

To achieve this aim, several analysis methodologies have been proposed in recent years [3,8-10]. In particular, great interest has been dedicated to the analysis of FHR variability (FHRV), which, like so for adults, could be a base for a more powerful, detailed and objective analysis, both in ante partum and in intra partum period [2,11-14]. The study of autonomic rhythms by FHR recordings may provide a sight into the foetal development of autonomic nervous system (ANS) [6].

Changes in FHR control, elicited by the ANS in response to foetal hypoxia, were reported in literature [3, 15]. A UC is a strong compressive stimulus; it provokes an acute hypoxic stress to the foetus and generally elicits reactions in the FHR. It is well known that FHR decelerations are often associated with UC and that their characteristics are of great interest for physicians [4]. Moreover, although the FHR is subject to numerous influences, UC is the only input which can be externally monitored [7]. Interest in studying UC reactions is also outlined by recent studies in which UC were elicited by an oxitocin challenge test to explore the consequent blood flow changes $[2,16,17]$. In conclusion, it is worth investigating FHRV modifications, which reflect reactions of foetal ANS to UC, in order to have more comprehensive information about the insult and the foetal ability to withstand it. This could provide additional and objective information about foetal health and then support clinical diagnosis.

Concerning FHRV estimation, even if, as it is known, it can be analysed both in time domain and in frequency domain, the power spectral density (PSD) seems to be the index that best recovers all the information present in the heart rate (HR) series [18]. Spectral analysis provides a tool for quantifying rather small changes in FHRV in response to internal or external stimuli that may remain undetected if only visual interpretation of FHR tracings is used. Among most common methods employed to estimate PSD, parametric and non-parametric, we can mention Short-time Fourier transform (STFT); Auto Regressive methods (AR); Fast Recursive least square algorithms (RLS) [2,12,14,19], wavelet transform [20] and Lomb method [21].

Supported by previous results [1,22], this study aimed to analyse more in depth spectral modifications in the FHRV signal (by means of STFT) in response to UC for healthy foetuses, which may help in the understanding of specific foetal reactivity, capability and modality of foetal compensation to hypoxic stress, by using the natural disturbance caused by UC. In particular, considering physiological cases, we would highlight the specific modification pattern of FHRV power spectrum, here regarded as ANS response. In future works, this pattern could be compared to patterns corresponding to pathological conditions in order to define a new classification criterion.

\section{METHODS}

\subsection{Data Collection}

CTG were recorded during routine foetal monitoring, in an Italian public hospital, from 35 healthy pregnant women (singleton pregnancies), close to delivery (33-42 gestation weeks), who did not take drugs and having no known genetic malformations; subjects laid down in a rest position. In line with clinical practice, CTG signals lasting less than $20 \mathrm{~min}$ or excessively noisy signals were excluded from our database (at the moment populated by about 600 CTG). 35 CTG recordings were gathered for this study, 3 intra partum and the others with evident UC. On average, CTG recordings have a duration of about 30 minutes. At birth, Apgar scores, birth weights and other information were collected in order to involve in the analysis only CTG regarding healthy foetuses: in particular, enrolled infants had Apgar scores $>=7$ at 1 st minute and $>=9$ at 5 th minute, birth weights (ranging from 2.7 to $4.25 \mathrm{Kg}$ ) appropriate for the gestational age and no one needed neonatal intensive care unit treatment.

Cardiotocographic signals were acquired using HP-135x or Sonicaid cardiotocographs, equipped with an ultrasound Doppler probe to detect FHR signals (measured in beats per minute-bpm) and a pressure transducer to record UC signals (measured in $\mathrm{mmHg}$ ). Both probes were placed upon maternal abdomen.

In HP cardiotocographs, FHR and UC signals are internally stored at $4 \mathrm{~Hz}$ (corresponding to a sampling interval of $250 \mathrm{~ms}$ ). On the contrary, in Sonicaid cardiotocograph, FHR and UC signals are unevenly stored. Both devices provide a three-level signal which indicates the 'quality' of the received Doppler signal, which can result optimal, acceptable or insufficient (the latter corresponding to signal loss). In both cases, recorded data are transferred to the output serial port of the device that was connected to a laptop PC through a serial (RS232) connection.

\subsection{Signal Selection}

CTG recordings with evident UC were chosen for the analysis; as done in previous works of the authors [1,22]. UC were selected respecting specific criteria in order to reduce the physiological variability and to achieve a sort of uniformity for the UC stimuli. In particular, only 
uterine contractions of pronounced amplitude (at least 40 $\mathrm{mmHg}$ with respect to the resting tone), isolated (at least $130 \mathrm{~s}$ must elapse between the end and the start of two subsequent contractions), corresponding to good FHR and UC signal quality were considered for the analysis. About $100 \mathrm{UC}$, compliant with the above-mentioned specifications were enrolled in the analysis.

In order to carry out a quantitative comparison between the FHRV power spectrum modifications related to UC with respect to a reference condition, two kinds of time segments of the same length (231 samples, about 57 s) were selected; let us call them: 'reference-segments', chosen before the UC onset and 'UC-segments', chosen in correspondence of the UC (slightly retarded with respect to the UC apex) (please, refer to the previous publication for more detailed information [1]).

Figure 1 offers an example of CTG signals and chosen segments.

\subsection{Pre-Processing}

Before FHR signal processing, it is worth mentioning that, because CTG is acquired in a clinical setting, it is subject to specific noises; for example, the loss of probe contact can temporarily interrupt the recording. Moreover, FHR signals are intrinsically uneven series; each FHR value is computed as inverse of the time between two consecutive $\mathrm{R}$ waves, so that FHR values are available only when new heart beats occur. To obtain evenly sampled series, some commercial cardiotocographs (e.g. HP-135x) use a zero-order interpolation, that is each sample is held constant until the next heart beat occurs. This is an efficient solution for FHR time-domain analyses (accelerations and/or decelerations detection, etc) but can introduce alterations in the FHR power spectrum $[18,22]$. To overcome these limitations, CTG recordings were pre-processed, by means of an algorithm previously developed by the authors, in order to select reliable FHR segments, to eliminate possible artifacts related to the Doppler technique and, only when necessary, to get rid of the zero-order interpolation [23-25].

\subsection{FHRV Time-Frequency Analysis}

According to literature and previous works [1,7,22], we considered the FHR power spectrum mainly composed of a DC component (average of the FHR), a very low frequency (VLF) band (0-0.03 Hz) and FHR variability (FHRV) at higher frequencies. Therefore, FHRV signals were obtained evaluating (and then subtracting) components at lower frequencies by means of a smoothing cubic spline.

After that, because of the non-stationary behaviour of the FHRV signal, a time-varying frequency analysis by means of STFT had been carried out considering sliding Hamming windows of 128 samples (corresponding to 32 s) and using 99\% overlap (window length was chosen according to literature [26]).
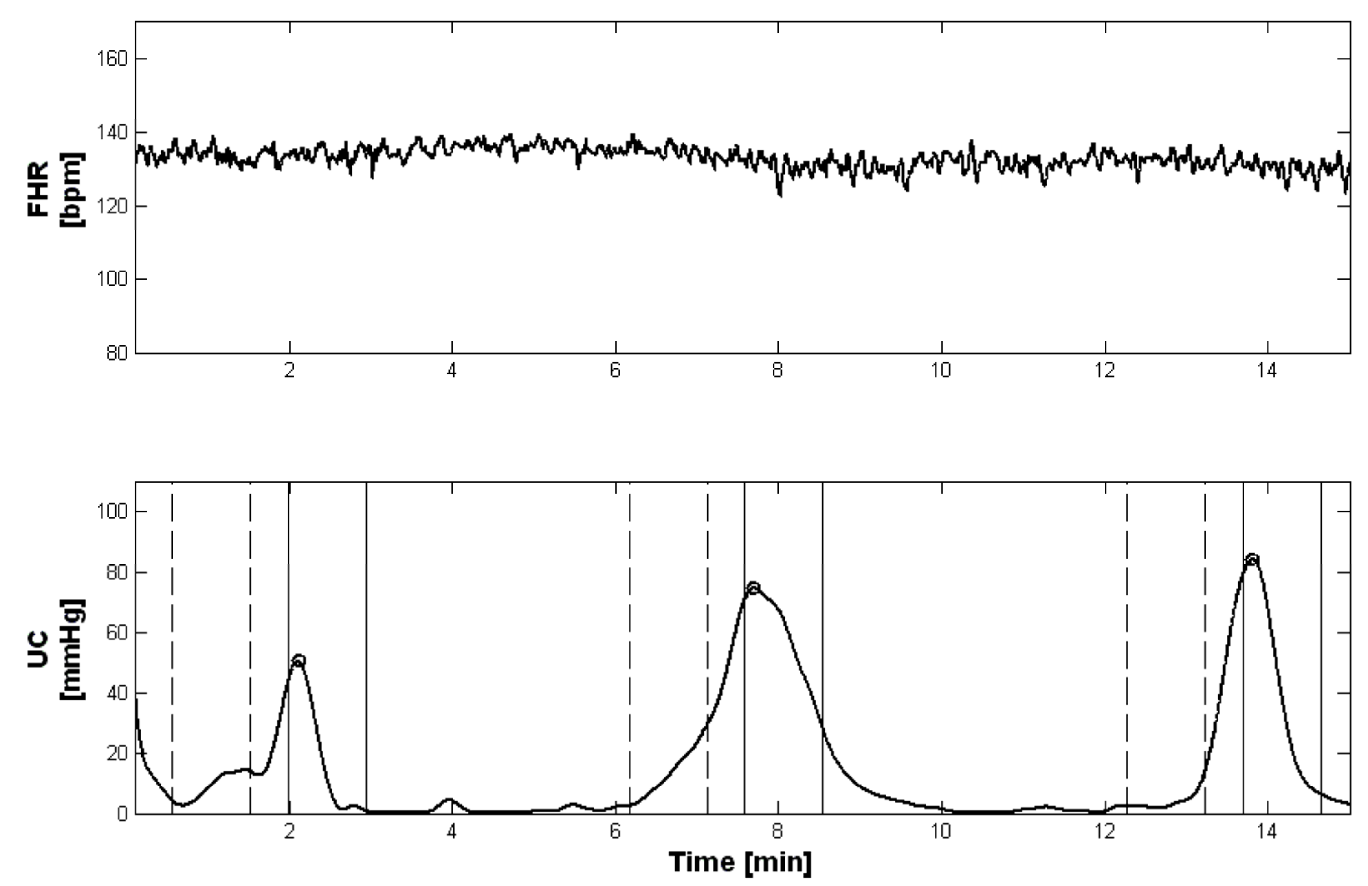

Figure 1. Example of CTG recording (from the top, FHR and UC signals) during labour, subject \#12-week $40^{\text {th }}$. It is possible to recognise three UC, which were selected according to the required criteria. Couples of vertical dashed lines represent start and end of reference segments and vertical solid lines represent start and end of UC segments. 
Finally, to concisely describe spectral modifications against time, the power associated with LF $(0.03-0.2 \mathrm{~Hz})$ and $\mathrm{HF}(0.2-1 \mathrm{~Hz})$ bands was computed, for each time instant, PLF ( $\mathrm{t})$ and $\operatorname{PHF}(\mathrm{t})$, as expressed by [1]:

$$
\begin{aligned}
& P_{L F}(t)=\frac{1}{T} \int_{0.03}^{0.2}|S(f, t)|^{2} d f \\
& P_{H F}(t)=\frac{1}{T} \int_{0.2}^{1}|S(f, t)|^{2} d f
\end{aligned}
$$

where, $\mathrm{S}(\mathrm{f}, \mathrm{t})$ represents the time-varying spectral estimation of the FHRV signal and $\mathrm{T}$ is the time interval considered [1].

In order to highlight a common foetal ANS response to UC in a physiological situation, we also performed an average of LF and HF power signals (computed both for $\mathrm{UC}$ and references segments).

\subsection{FHRV Frequency Content}

To further characterise PSD modifications of FHRV signals related to UC, we carried out an analysis to detect, at each time instant, the maximum frequency bin contained in the signal's spectrum. To this aim, we used the "Modified Crossing Threshold Method", based on D'Alessio's algorithm $[22,27,28]$. The method considers that the tail of the spectrum gives information on the level of noise present in the signal, since white noise is equally spread over all frequency. An estimation of the noise made in the tail of the spectrum is then used for setting a threshold. The magnitude of each bin of the spectrum is compared with the threshold and when the magnitudes of two successive bins are higher than the threshold, the first bin is considered as the maximum frequency bin. We evaluated the noise level using the bins from 14 to 32 of a 128 FFT array (corresponding to the frequency range from about $0.4 \mathrm{~Hz}$ to $1 \mathrm{~Hz}$ ). The selected frequency range to evaluate the noise depends on the method used in evaluating the FHR and its spectrum array [27], which could substantially modify the far tail of the spectrum. The algorithm threshold is computed multiplying this estimation of noise for an integer factor; we heuristically chose a value of 5 , which means that the probability value for which a sample crosses the threshold is less than $1 \%$ in presence of only noise [28].

\subsection{Statistic Analysis}

A Student's $t$-test was employed to check the statistic separation between the analysed FHRV spectral populations (power in the different bands and frequency content of UC-segments and reference-segments; levels of statistical significance were always set at $\mathrm{p}$ value $<$ 0.01 ).

For power estimation in HF band, we chose the fixed range $0.2-1 \mathrm{~Hz}$, without considering the computed vari- able maximum frequency bin, in order to take into account noise contribution both for UC and ref segments.

\section{RESULTS}

As an example, Figure 2 reports a spectrogram Figure 2(a) of a FHRV time-frequency distribution, obtained by STFT method, together with the corresponding CTG signal (FHR in Figure 2(c) and UC in Figure 2(b)).

As illustrated by Figure 2(a) the FHRV power increases in correspondence of UC.

It is worth noting that this increase does not correspond, in the time domain, to a clear modification of the floating-line (id est, in the FHR signal there are no alterations, such as accelerations or decelerations, that could justify the power increase).

Furthermore, to present concise results, obtained average powers of LF and HF bands of FHRV power spectrum, estimated both for selected UC segments and reference segments, are reported in Table 1.

It is possible to note that average powers corresponding to UC-segments are higher than average powers corresponding to reference-segments. Moreover, UC-segments population resulted significantly different compared with reference-segments population for both $\mathrm{FH}$ $\mathrm{RV}$ power spectrum bands ( $t$-test).

Concerning the analysis of FHR frequency content, results obtained by means of Modified Crossing Threshold Method highlighted that an enlargement of the band (shift to a higher value of signal maximum frequency bin) corresponds to the power increase of FHRV PSD. The following Figure 3 shows an example of obtained results about the comparison between the power increase and the correspondent shift of signal maximum frequency bin.

The average behaviour has been studied also in this case and obtained results are reported in Table 2.

Our average results highlighted, in correspondence of the $\mathrm{UC}$, a percentage increase of the band, computed as

$\frac{\text { UC seg. value - REF seg. value }}{\text { REF seg. value }} \times 100$, of about $27 \%$.

Also in this case, UC-segments population resulted significantly different compared with reference-segments population ( $t$-test).

\section{DISCUSSIONS}

Cardiotocography is an established part of daily obstetric practice, to monitor foetal health, mostly in the last weeks of gestation. Clinicians regularly monitor FHR and UC for signs of at-risk (or compromised) foetal conditions. During labour, to assess foetal reactivity, attention is focused on FHR alterations (such as decelerations) in correspondence to UC. 

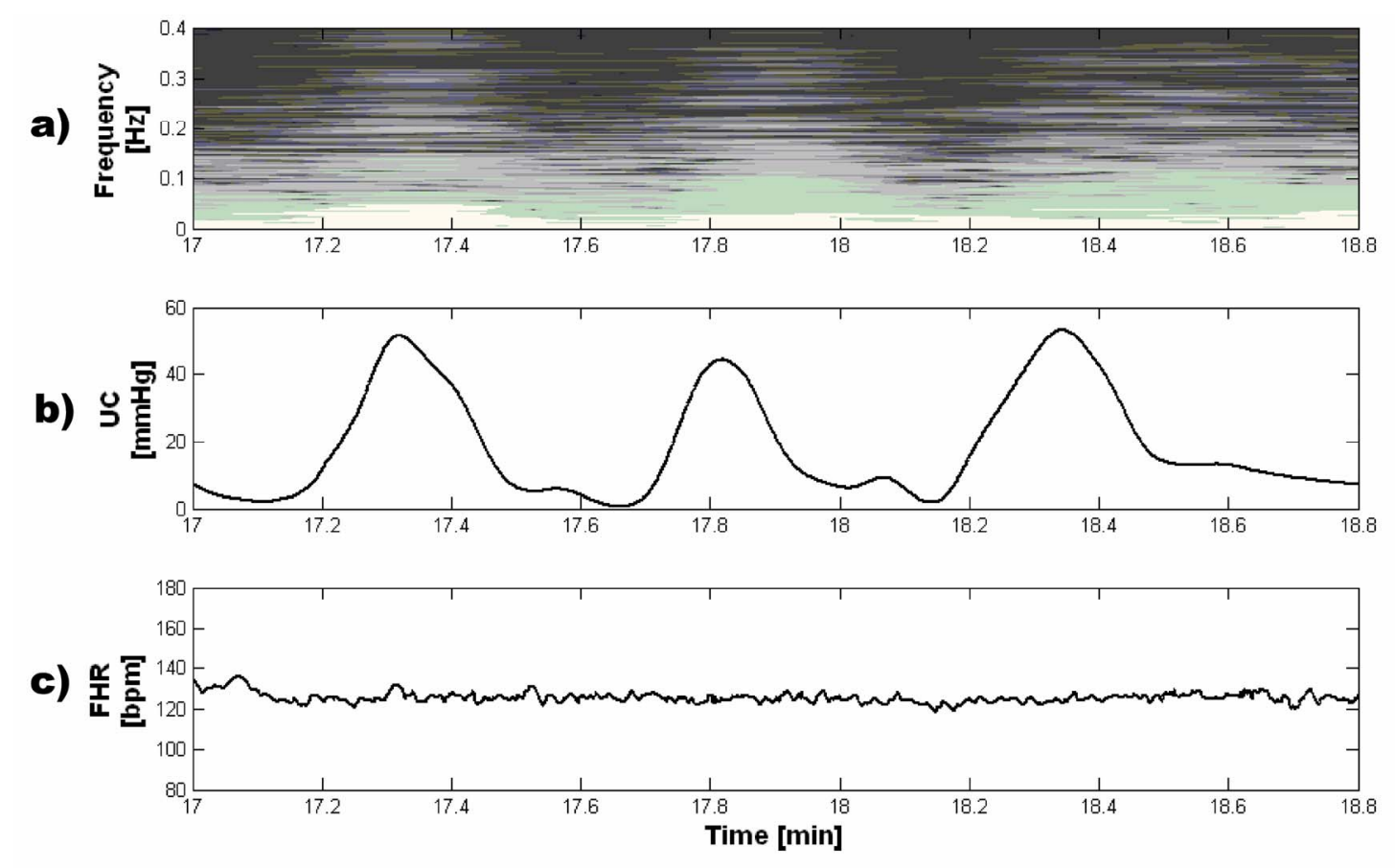

Figure 2. Example of FHRV spectral modifications in correspondence of UC. From the top, spectrogram, evaluated by means of STFT, and CTG signal of the subject \#24 (UC in 2(b), FHR in 2(c)).

Table 1. Powers in LF and HF bands, computed by means of STFT, are reported for the UC and reference segments in $\mathrm{bpm}^{2}$. Reported values represent average results computed on 108 segments, in brackets we report the standard deviation.

\begin{tabular}{lll}
\hline & UC segments $\left[\mathbf{b p m}^{2}\right]$ & REF segments [bpm $\left.{ }^{2}\right]$ \\
\hline $\begin{array}{l}\text { Power of LF } \\
\text { band }\end{array}$ & $459.44(205.36)$ & $183.72(113.94)$ \\
$\begin{array}{l}\text { Power of HF } \\
\text { band }\end{array}$ & $164.15(85.36)$ & $81.53(62.15)$ \\
\hline
\end{tabular}

CTG usefulness is undoubted; nevertheless, there is, still nowadays, substantial intra- and inter-observer variation in the assessment of FHR patterns, due mainly to the visual inspection of CTG, which can lead to intervention when it is not required or lack of intervention when it is. Hence, several analysis methodologies (in time domain, in frequency domain, with semi-automatic software which compute specific time-domain parameters, etc.) were proposed in recent years to improve reliability and objectivity of CTG signals interpretation $[2,3]$.

This is still insufficient to certainly identify suspect or ambiguous conditions. So, great interest was dedicated to the FHRV. It is commonly accepted that the use of a convenient technique for measuring and displaying beat to beat fluctuations is of value for estimating the maturation of ANS and the integrity of the nervous control of heart rate [29]. In particular, frequency analysis of FHRV could be a useful, additional tool $[8,12,30]$ both in ante partum and in intra partum period.

A careful surveillance has to be dedicated to the intra partum period; in fact, while labour is of short duration in comparison to pregnancy, this period is of great risk for the foetus [31]. Intra partum stress can provoke adaptive changes in foetal metabolic process that can impact the future health of newborn. [6]. Therefore, clinicians regularly check FHR and UC to try to identify foetal distress symptoms and adapt the extracting procedure for signs of at risk foetuses. In particular FHR alterations in correspondence to UC are evaluated, to assess foetal reactivity. However, it is well known that there is still controversy over the interpretation of different FHR patterns and that objective clinical criteria to recognise foetal distress by CTG data are still poorly defined, especially during labour [32] and no clear conclusions are available so far. Positive predictive value of abnormal intra partum FHR patterns for foetal acidemia is only around $30 \%$ [15], whereas detection of foetal distress, early in labour, may significantly improve newborn's health. Besides, literature regarding intra partum CTG is much less rich than that about ante partum CTG, mainly due to registration difficulties. Therefore, it is important to try to obtain more reliable and objective methods for CTG interpretation and for neonatal outcome prediction [16,17,33-37].

In this scenario, analysis of FHRV can provide additional, useful information related to the foetal ANS control of the heart and its compensation capability. Analo- 

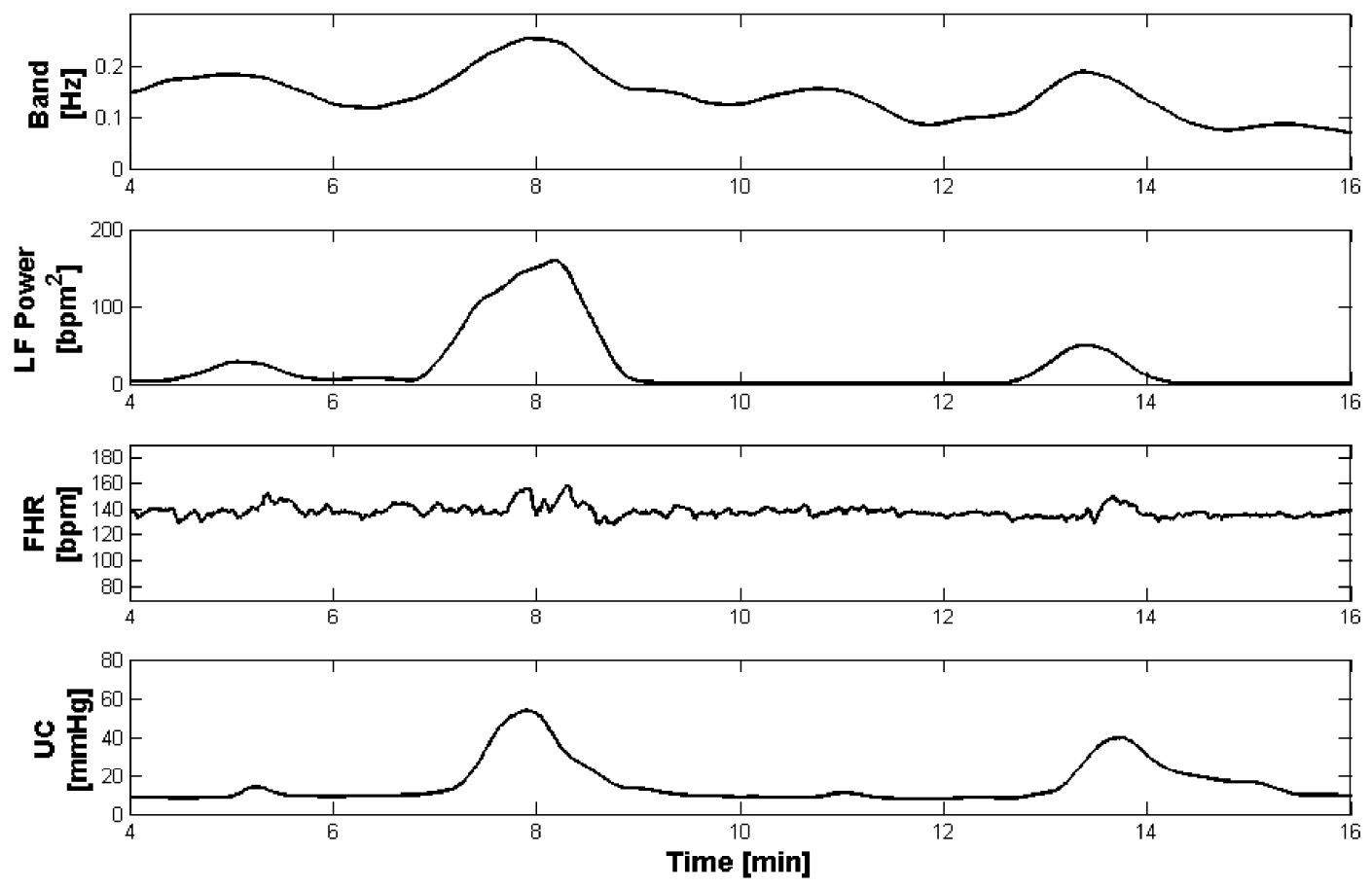

Figure 3. Example of FHRV spectral modifications. From the top, frequency content, evaluated by means of the Modified Crossing Threshold Method, power of the LF band, and CTG signal of the subject \#18.

Table 2. Maximum frequency bin contained in the analyzed signals both for UC segments and reference segments. Reported values represent average results computed on 108 segments, in brackets we report the standard deviation.

\begin{tabular}{lll}
\hline & UC segments [Hz] & REF segments [Hz] \\
\hline $\begin{array}{l}\text { Maximum frequency } \\
\text { bin }\end{array}$ & $0.218(0.064)$ & $0.172(0.045)$ \\
\hline
\end{tabular}

gously as for adults, specific stimuli can alter heart autonomic regulation and in turn generate specific modifications in the HR, particularly evident in frequency domain. Indeed, a UC is a strong compressive stimulus [38] (intra-uterine pressure can become four times stronger than basal pressure) that severely solicits the immature foetal ANS. This stress causes reactions in the FHR; one of the most evident is a FHR deceleration that often is associated with a UC, which is an important sign for physicians. Therefore, a more detailed study of the reaction of foetal ANS to UC, such as FHRV spectral modifications analysis, may help in the understanding of specific foetal reactivity, capability and modality of foetal compensation to hypoxic stress.

This work presents a study to investigate spectral modifications of the FHRV in response to the external stimulus represented by UC, for healthy foetuses, in order to find, during labour, possible predictive information about risky foetal conditions, before foetuses become injured.
We did not consider the gestational age (related to ANS maturation), even if it is well known that considerably affects the foetal hemodynamic responses to stimuli and distress, because weeks of gestation, in all our recordings, were in a range where it is possible to disregard this factor as an additional cause of considerable FHR changes $[18,39,40]$.

Clinical intra partum UC and FHR are very noisy signals prone to frequent sensor disturbances; however, despite these conditions, the segment length required for frequency analysis, as proposed in this work, is short enough to overcome this problem.

Our results showed that a FHRV power spectrum modification can be observed in response to UC stimulus. In particular, a significant increase of the average power (confirmed by a $t$-test, $\mathrm{p}<0.01$ ) during UC-segments with respect to reference-segments is noted.

Moreover, we observed a shift to higher values of the maximum frequency contained in the signal in correspondence of the power increase. So that, we can conclude that the power increase is not due to a specific band enlargement but is spread over all frequencies.

By literature it is known that, in general, a large variability reflects a healthy ANS and also chemoreceptors, baroreceptors and cardiac responsiveness; while foetal hypoxia, congenital heart anomalies and stress, cause a decreased variability $[3,4,41]$. So, in healthy foetuses, 
we expected an evident modification in FHRV frequency characteristics corresponding to a good capability of reaction to UC stimulus.

Obtained results, according to that finding, should indicate a foetal reactivity (in terms of FHRV power spectrum modifications with respect to the rest condition) to mechanical compressive stimulus represented by UC for healthy foetuses. Therefore, such spectral modifications, being a sign of ANS reaction, could represent additional, objective information about foetal reactivity and in turn about foetal health during labour.

In a future perspective, it should be very interesting to analyse not only the average behaviour of FHR spectral modifications but also its trend during labour course in order to evaluate the individual status of a foetus and possibly to set an alarm threshold. Since foetal response is probably due to number and frequency as well as intensity of UC, it is important to establish a criterion for an objective and standardised measure of the stimulus intensity. In fact, let us remind that in cardiotocography only a relative measure of UC is known.

However, these issues deserve a more detailed and exhaustive analysis, mainly involving problematic pregnancies and cases of ascertained foetal distress, in order to, considering foetal health situations as point of reference, to recognise specific spectral characteristics to distinguish foetal well-being and foetal distress in order to propose such methodology in daily clinical practice.

\section{CONCLUSIONS}

The Variability of the Foetal Heart Rate around its baseline provides extremely significant information concerning the cardiac and ANS activities and their functional development during pregnancy up to labour. Our results demonstrated important modifications in the PSD of FHRV signals related to physiological stimulus represented by UC, both as power and frequency content, proving that the FHRV time-frequency analysis could be a very useful tool for a more objective and depth evaluation of the foetal health. We would not expect to find such modifications in cases of foetal distress; therefore, if these results will be confirmed by the study of FHRV signals recorded during risky pregnancies, information about the capability of a foetus to react to stress during labour course could be obtained by means of this approach.

\section{REFERENCES}

[1] Romano, M., Bifulco, P., Cesarelli, M., Sansone, M. and Bracale, M. (2006) Fetal heart rate power spectrum response to uterine contraction. Medical \& Biological Engineerin \& Computing, 44(3), 188-201.

[2] Cesarelli, M., Romano, M. and Bifulco, P. (2009) Com- parison of short term variability indexes in cardiotocographic foetal monitoring. Computers in Biology and Medicine, 39(2), 106-118.

[3] Signorini, M.G., Magenes, Cerutti, G.S. and Arduini, D. (2003) Linear and nonlinear parameters for the analysis of fetal heart rate signal from cardiotocographic recordings. IEEE Transaction on Biomedical Engineering, 50(3), 365-375.

[4] Sweha, A. and Hacker, T.W. (1999) Interpretation of the electronic fetal heart rate during labor. American Academy of Family Physician, 59(9), 2487-2500.

[5] Rizzo, N., Accorsi, P., Baronciani, D., et al., "La sorveglianza del benessere fetale in travaglio di parto. Linea guida basata su prove di efficacia Regione Emilia Romagna, Progetto n. 3.

[6] Hoyer, D., Heinicke, E., Jaekel, S., Tetschke, F., Paolo, D.P., Haueisen, J., Schleußner, E. and Schneider, U. (2009) Indices of fetal development derived from heart rate patterns. Early Human Development, 85, 379-386.

[7] Philip, A., Warrick, E.F., Hamilton, D.P. and Robert, E.K. (2009) Identification of the dynamic relationship between intra-partum uterine pressure and fetal heart rate for normal and hypoxic fetuses. IEEE Transactions on Bio-Me- dical Engineering, 56(6), 1587-1597.

[8] Martin, C.B. (1982) Physiology and clinical use of fetal heart rate variability. Clinics in Perinatology, 9(2), 339352.

[9] Dawes, G.S., Meir, Y.J. and Mandruzzato, G.P. (1994) Computerized evaluation of fetal heart-rate patterns. Journal of Perinatal Medicine, 22(6), 491-499.

[10] Romano, M., Cesarelli, M., Bifulco, P., Sansone, M. and Bracale, M. (2002) Development of an algorithm for homogeneous FHR signals identification. Proceedings of Embec'02 2nd European Medical and Biological Engineering Conference, December 2002, Vienna, Austria; II: 1542.

[11] Sibony, O., Fouvillot, J.P., Benaoudia, M., Benhalla, A., Oury, J.F., Sureau, C. and Blot, P. (1994) Quantification of the heart rate variability by spectral analysis of fetal well-being and fetal distress. European Journal of $\mathrm{Ob}$ stetrics \& Gynecology and Reproductive Biology, 54(2), 103-108

[12] Cerutti, S., Civardi, S., Bianchi, A., Signorini, M.G., Ferrazzi, E. and Pardi, G. (1989) Spectral analysis of antepartum heart rate variability. Clinical Physics and Physiological Measurement, 10(Suppl B), 27-31.

[13] Divon, M.Y., Muska, Y., Platt, L.D. and Paldi, E. (1984) Increased beat to beat variability during uterine contractions: A common association in uncomplicated labor. American Journal of Obstetrics and Gynecology, 149(8), 893896.

[14] Romano, M., Bracale, M., Cesarelli, M., Campanile, M., Bifulco, P., Falco, M.D., Sansone, M. and Lieto, A.D. (2005) Antepartum cardiotocography: A study of fetal reactivity in frequency domain. Computers in Biology and Medicine, 36(6), 619-633.

[15] Geijn, H.P. (1996) Developments in CTG analysis. Baillieres Clin Obstet Gynaecol, 10(2), 185-209.

[16] Gudmudsson, S. and Olofsson, P. (2004) Acute changes of cerebral venous blood flow in growth-restricted human fetuses in response to uterine contractions. Ultra- 
sound Obstet Gynecol, 24(5), 516-521.

[17] Li, H., Gudmundsson, S. and Olofsson, P. (2003) Acute increase of umbilical artery vascular flow resistance in compromised fetuses provoked by uterine contractions. Early Human Development, 74(1), 47-56.

[18] Laguna, P., Moody, G.B. and Mark, R.G. (1998) Power spectral density of unevenly sampled data by least-square analysis: performance and application to heart rate signals. IEEE Transactions on Biomedical Engineering, 45(6), 698-715.

[19] Pola, S., Macerata, A., Emdin, M. and Marchesi, C. (1996) Estimation of the power spectral density in nonstationary cardiovascular time series: Assessing the role of the time-frequency representation (TFR). IEEE Transactions on Biomedical Engineering, 43(1), 46-59.

[20] Salamalekis, E., Thomopoulos, P., Giannaris, D., Salloum, I., Vasios, G., Prentza, A. and Koutsouris, D. (2002) Computerised intrapartum diagnosis of fetal hypoxia based on fetal heart rate monitoring and fetal pulse oximetry recordings utilising wavelet analysis and neural networks. BJOG: An International Journal of Obstetrics and Gynaecology, 109(10), 1137-1142.

[21] Cesarelli, M., Romano, M., Ruffo, M., Bifulco, P., Pasquariello, G. and Fratini, A. (2009) PSD modifications of FHRV due to CTG storage rate. Proceedings of 9th International Conference on Information Technology and Applications in Biomedicine, Larnaca, Cyprus.

[22] Romano, M., Cesarelli, M., Bifulco, P., Ruffo, M., Fratini, A. and Pasquariello, G. (2009) Time-frequency analysis of CTG signals. Current Development in Theory and Applications of Wavelets, 3(2), 169-192.

[23] Cesarelli, M., Romano, M., Bifulco, P., Fedele, F. and Bracale, M. (2007) An algorithm for the recovery of fetal heart rate series from CTG data. Computers in Biology and Medicine, 37(5), 663-669.

[24] Romano, M., Cesarelli, M., Bifulco, P., Sansone, M. and Bracale, M. (2003) Study of fetal autonomous nervous system's response by means of FHRV frequency analysis. 1st International IEEE EMBS Conference on Neural Engineering, Capri, Italy, 399-402.

[25] Hieftje, G.M., Bistroff, R.I. and Lim, R. (1973) Application of correlation analysis for signal-to-noise enhancement in flame spectrometry. Analytical chemistry, 45(2), 253-258

[26] Karin, J., Hirsch, M., Sagiv, C. and Akeselrod, S. (1992) Fetal autonomic nervous system activity monitoring by spectral analysis of heart rate variations. IEEE Proceedings of Conference on Computers in Cardiology, 479-482.

[27] Moraes, R., Aydin, N. and Evans, D. (1995) The performance of three maximum frequency envelope detection algorithms for Doppler signals. Journal of Vascular Investigation, 1(3), 126-134.

[28] Alessio, T.D. (1985) Objective algorithm for maximum frequency estimation in Doppler spectral analysers. Medical \& Biological Engineering \& Computing, 23(1), 63-68.
[29] David, M., Hirsch, M. and Akeselrod, S. (2006) Maturation of fetal cardiac autonomic control as expressed by fetal heart rate variability. IEEE Proceedings of Conference on Computers in Cardiology, 901-904.

[30] Oppenheimer, L.W. and Lewinsky, R.M. (1994) Power spectral analysis of fetal heart rate. Baillère's Clinical Obstetrics and Ginecology, 8(3), 643-661.

[31] Logier, R., Jonckheere, J.D., Jeanne, M. and Matis, R. (2008) Fetal distress diagnosis using heart rate variability analysis: design of a high frequency variability index. IEEE Proceedings of 30th Annual International IEEE EMBS Conference Vancouver, British Columbia, 47284731.

[32] Cao, H., Lake, D.E., Chisholm, C.A., Ferguson, J.E., Grifin, M.P. and Moorman, J.R. (2003) Toward quantitative monitoring of human cardiotocography during labor. Proceedings of the 25th Annual International Conference of the IEEE EMBS, Cancun, Mexico.

[33] Salamalekis, E., Vitoratos, N., Loghis, C., Panayotopoulos, N., Kassanos, D. and Creatsas, G. (1999) Evaluation of fetal heart rate patterns during the second stage of labor through fetal oximetry. Gynecologic and Obstetric Investigation, 48, 151-154.

[34] Zimmer, E.Z., Paz, Y., Copel, J.A. and Weiner, Z. (1998) The effect of uterine contractions on intrapartum fetal heart rate analyzed by a computerized system. American Journal of Obstetrics and Ginecology, 178(3), 436-440.

[35] Jensen, O.H.R. and Narverud, G. (1994) Fetal heart rate decelerations and umbilical cord blood gas values. European Journal of Obstetrics \& Gynecology and Reproductive Biology, 53(2), 103-106.

[36] Padhye, N.S., Duan, Z. and Verklan, M.T. (2004) Response of fetal heart rate to uterine contractions. Proceedings of the 26th Annual International Conference of the IEEE EMBS, San Francisco, 3953-3955.

[37] Kodama, Y., Sameshima, H., Ikeda, T. and Ikenoue, T. (2009) Intrapartum fetal heart rate patterns in infants ( $\geq 34$ weeks) with poor neurological outcome. Early $\mathrm{Hu}$ man Development, 85(4), 235-238.

[38] Goeschen, K. (1998) Cardiotocografia pratica. Roma, V edizione, CIC Edizioni Internazionali, Roma

[39] Rantonen, T., Ekholm, E., Siira, S., Metsala, T., Leino, R., Ekblad, U. And Valimaki, I. (2001) Periodic spectral components of fetal heart rate variability reflect the changes in cord arterial base deficit values: A preliminary report. Early Human Development, 60(3), 233-238.

[40] Ohta, T., Okamura, K., Kimura, Y., Suzuki, T., Watanabe, T., Yasui, T., Yaegashi, N. and Yajima, A. (1999) Alteration in the low-frequency domain in power spectral analysis of fetal heart beat fluctuations. Fetal Diagnosis and Therapy, 14(2), 92-97.

[41] .Geijn, H.P., Jongsma, H.W., Haan, J. and Eskes. T.K. A.B. (1980) Analysis of heart rate and beat-to-beat variability: Interval difference index. American Journal of Obstetrics and Ginecology, 138(3), 246-252. 